


\title{
MEASURE ALGEBRAS OF SEMILATTICES WITH FINITE BREADTH
}

\author{
J. D. LAWSON, J. R. LIUKKONEN \\ AND M. W. MisLOVE
}

The main result of this paper is that if $S$ is a locally compact semilattice of finite breadth, then every complex homomorphism of the measure algebra $M(S)$ is given by integration over a Borel filter (subsemilattice whose complement is an ideal), and that consequently $M(S)$ is a $P$-algebra in the sense of S. E. Newman. More generally it is shown that if $S$ is a locally compact Lawson semilattice which has the property that every bounded regular Borel measure is concentrated on a Borel set which is the countable union of compact finite breadth subsemilattices, then $M(S)$ is a $P$-algebra. Furthermore, complete descriptions of the maximal ideal space of $M(S)$ and the structure semigroup of $M(S)$ are given in terms of $S$, and the idempotent and invertible measures in $M(S)$ are identified.

In earlier work Baartz and Newman have shown that if $S$ is the finite product of totally ordered locally compact semilattices, then every complex homomorphism is given by integration over a Borel subsemilattice whose complement is an ideal [1, Th. 3.15], and consequently, the structure semigroup of $M(S)$ in the sense of Taylor [10] is itself a semilattice [9, Th. 3]. In both papers it is shown that such results do not hold for the infinite dimensional cube $S=I^{\omega}$, and Newman conjectures that what is needed for these results to hold is a "finite dimensionality" condition. In this paper it is shown that these results hold provided the locally compact semilattice in question has "finite breadth"; i.e., satisfies a finite dimensionality condition familiar from the theory of compact semilattices.

The paper is organized as follows. Section 1 contains generalities on semilattices and the notion of breadth. Section 2 is devoted to the proof of our main result for finite breadth semilattices. In $\S 3$ we discuss the extension of these results to a more general setting and give examples to show how our hypotheses differ from those of Newman.

1. Semilattices. A semilattice is a commutative idempotent semigroup. We may also (equivalently) describe a semilattice as a partially ordered set in which every two elements have a greatest lower bound. Thus the product of two elements is their greatest 
lower bound. The reader should note that this convention differs from that in $[1,9]$, in which the product of two elements in a semilattice is viewed as their least upper bound.

A semilattice on a Hausdorff space $S$ is a topological (semitopological) semilattice if the multiplication function which sends $(x, y)$ to $x y$ from $S \times S$ to $S$ is jointly (separately) continuous. It is known that a compact semitopological semilattice is actually a topological semilattice [8].

If $S$ is a semilattice and $A \subset S$ we define the upper and lower sets of $A$ by

$$
\uparrow A=\{y \in S: x \leqq y \text { for some } x \in A\}
$$

and

$$
\downarrow A=\{z \in S: z \leqq x \text { for some } x \in A\} .
$$

For singleton sets we adopt the notation $\uparrow x$ and $\downarrow x$ instead of $\uparrow\{x\}$ and $\downarrow\{x\}$. We call a subset $I$ of $S$ an $i d e a l$ if $\downarrow I=I$. Equivalently $I$ is an ideal if $x \in S, y \in I$ implies $x y \in I$. A subset $F$ of $S$ is a filter if $\uparrow F=F$ and $F$ is a subsemilattice of $S$. Note that a subsemilattice $F \subseteq S$ is a filter if and only if $S \backslash F$ is an ideal of $S$.

If $A$ is a nonempty subset of a semilattice $S$ we denote the greater lower bound of $A$ by $\wedge A$ ( $\wedge A$ exists for all finite sets $A$, and also for all infinite sets if $S$ is a compact topological semilattice). A fiinite set $A$ is said to be meet-irredundant if $\wedge A<\wedge B$ for any proper subset $B$ of $A$. A semilattice $S$ is said to have breadth $n$ (denoted br $(S)=n$ ) if $n$ is the greatest cardinality of the meetirredundant subsets of $S$. Equivalently $S$ has breadth $n$ if and only if $n$ is the smallest integer such that any finite subset $J$ of $S$ of cardinality $m>n$ has a subset $L$ of cardinality $n$ such that $\wedge J=\wedge L$, and this is equivalent to $n$ being the smallest integer such that any finite subset $J$ of cardinality $n+1$ has a subset $L$ of cardinality $n$ such that $\wedge J=\wedge L$. We adopt the convention that a singleton semilattice has breadth 0 .

A subset $A$ of a semilattice $S$ is bounded above if there exists $p \in S$ such that $p \geqq \alpha$ for all $a \in A$. The bounded breadth of $S$ is $n$ (denoted $\operatorname{bbr}(S)=n$ ) if $n$ is the greatest cardinality of any meet-irredundant set bounded above.

Proposition 1.1. Let $S$ be a semilattice of finite breadth. Then $\operatorname{bbr}(S) \leqq \operatorname{br}(S) \leqq \operatorname{bbr}(S)+1$. If $S$ has an identity, then $\operatorname{bbr}(S)=\operatorname{br}(S)$.

Proof. Clearly bbr $(S) \leqq \operatorname{br}(S)$ and the two agree if $S$ has an 
identity (since in the latter case every set is bounded above). If $\left\{x_{1}, \cdots, x_{n}\right\}$ is a meet-irredundant set in $S$, let $y_{i}=x_{i} x_{n}$ for $i=1$, $\cdots, n-1$. Then it is straightforward to verify that $\left\{y_{1}, \cdots, y_{n-1}\right\}$ is a meet-irredundant set bounded by $x_{n}$. Hence it follows that br $(S) \leqq \operatorname{bbr}(S)+1$.

Proposition 1.2. Let $S$ be a topological semilattice such that $\operatorname{bbr}(S) \leqq n$, where $n \geqq 1$. If $I$ is a dense ideal of $S$, then for any subsemilattice $T$ contained in $S \backslash I$, bbr $(T) \leqq n-1$.

Proof. Let $x_{1}, \cdots, x_{n} \in T$ and let $b \in T$ such that $x_{i} \leqq b$ for $i=1, \cdots, n$, where $n \geqq 2$. Let $y_{\alpha}$ be a net in $I$ converging to $b$. Then $z_{\alpha}=y_{\alpha} b$ is a net in $I$ (since $I$ is an ideal) converging to $b b=b$. Since $\operatorname{bbr}(S) \leqq n$ for each $\alpha$ there exists $u_{\alpha} \in\left\{x_{1}, \cdots, x_{n}, z_{\alpha}\right\}=F_{\alpha}$ such that $\wedge F_{\alpha}=\wedge\left(F_{\alpha} \backslash\left\{u_{\alpha}\right\}\right)$. But $\wedge F_{\alpha} \in I$ since $z_{\alpha} \in I$; hence $u_{\alpha} \neq z_{\alpha}$ since $x_{i} \in T$ for $1 \leqq i \leqq n$ and $T$ is a subsemilattice. By picking subnets and renaming, we may assume $u_{\alpha}=x_{1}$ for each $\alpha$. Then $x_{1} \cdots x_{n}=\lim x_{1} \cdots x_{n} z_{\alpha}=\lim x_{2} \cdots x_{n} z_{\alpha}=x_{2} \cdots x_{n}$. Hence bbr $T \leqq n-1$.

Now suppose $n=1$. Let $x<b$ be two elements of $T$. Again let $\left\{z_{\alpha}\right\}$ be a net in $I$ such that $z_{\alpha} \leqq b$ for all $\alpha$ and $z_{\alpha} \rightarrow b$. Since $S$ has bounded breadth 1 and $I$ is an ideal, we see that $z_{\alpha}<x$ for all $\alpha$. Therefore $b=\lim z_{\alpha} \leqq x$, a contradiction. So $\operatorname{bbr}(T)=0$.

Proposition 1.3. Let $S$ be a topological semilattice and let $A=\left\{x_{1}, \cdots x_{n}\right\}$ be a meet-irredundant subset of $S$ of cardinality $n$. Then there exist open sets $U_{1}, \cdots, U_{n}$ such that $x_{j} \in U_{j}$ for $j=1$, $\cdots, n$ and if $y_{j} \in U_{j}$ for $j=1, \cdots, n$, then $\left\{y_{1}, \cdots, y_{n}\right\}$ is a meetirredundant set of distinct elements.

Proof. Suppose not. Then there exists a net $\left(y_{1 \alpha}, \cdots, y_{n \alpha}\right)$ converging to $\left(x_{1}, \cdots, x_{n}\right)$ in $\prod_{j=1}^{n} S$ such that for each $\alpha$, there exists $i, 1 \leqq i \leqq n$, such that $\bigwedge_{j=1}^{n} y_{j \alpha}=\wedge\left\{y_{j \alpha}: 1 \leqq j \leqq n, j \neq i\right\}$. By picking subnets and renumbering if necessary, we may assume that $y_{1 \alpha}$ is always the omitted. Then $\bigwedge_{j=1}^{n} x_{j}=\lim \bigwedge_{j=1}^{n} y_{j \alpha}=\lim \bigwedge_{j=2}^{n} y_{j \alpha}=$ $\bigwedge_{j=2}^{n} x_{j}$. However, this conclusion contradicts the hypothesis that $A$ is meet-irredundant.

In the following $T^{*}$ denotes the closure of $T$.

CoRollary 1.4. Let $T$ be a subsemilattice of finite breadth of a topological semilattice $S$. Then $\operatorname{br}(T)=\operatorname{br}\left(T^{*}\right)$.

Proof. Suppose $\operatorname{br}\left(T^{*}\right)=n$. Then there exists a meet-irredundant set $\left\{x_{1}, \cdots, x_{n}\right\}$ of cardinality $n$ in $T^{*}$. By Proposition 1.3 there exist 
open sets $U_{1}, \cdots, U_{n}$ with $x_{j} \in U_{j}$ for $1 \leqq j \leqq n$ such that if $y_{j}$ is chosen in $U_{j} \cap T$, then $\left\{y_{1}, \cdots, y_{n}\right\}$ is meet-irredundant. Hence $\operatorname{br}(T) \geqq n$. But since $T \subset T^{*}$, br $(T) \leqq n$.

Let $X$ be a compact Hausdorff space and let $P(X)$ denote the set of nonempty compact subsets of $X$. It is well known that $P(X)$ is a compact Hausdorff space when endowed with the topology of open sets generated by the subbasis

$$
N(U, V)=\{A \in P(X): A \subset U \text { and } A \cap V \neq \varnothing\}
$$

where $U$ and $V$ are arbitrary open subsets of $X$. A net $K_{\alpha}$ of compact subsets of of $X$ converges to $K \in P(X)$ if and only if $K=$ $\lim \sup K_{\alpha}=\lim \inf K_{\alpha}$. We call this topology the Vietoris topology.

Proposition 1.5. Let $K_{\alpha}$ converge to $K$ in $P(S)$ where $S$ is a compact semilattice. If each $K_{\alpha}$ is a compact topological subsemilattice of $S$ such that $\operatorname{bbr}\left(K_{\alpha}\right) \leqq n$, then $K$ is also a compact subsemilattice and $\operatorname{bbr}(K) \leqq n$. Hence the collection of compact subsemilattices of bounded breadth less than or equal to $n$ is a closed subset of $P(S)$.

Proof. It is known that $P(S)$ endowed with the operation $A B=$ $\{a b: a \in A, b \in B\}$ is a compact topological semigroup [4]. Since $K_{\alpha}$ converges to $K$, and $K_{\alpha} K_{\alpha}=K_{\alpha}$ for each $\alpha$, by continuity $K K=K$, i.e., $K$ is a subsemilattice.

Suppose $\operatorname{bbr}(K)>n$. Then there exists a meet-irredundant set $\left\{y_{1}, \cdots, y_{n+1}\right\}$ of distinct elements in $K$ and a $p \in K$ such that $y_{j} \leqq p$ for $1 \leqq j \leqq n+1$. By Proposition 1.3 there exist open sets $U_{1}, \cdots, U_{n+1}$ with $y_{j} \in U_{j}$ for all $j$ such that if a point is chosen from each $U_{j}$, the set of elements obtained is meet-irredundant. Pick by continuity of multiplication an open set $V, p \in V$, and open sets $V_{1}, \cdots, V_{n+1}, y_{j} \in V_{j}$ for $1 \leqq j \leqq n+1$, such that $V V_{j} \subset U_{j}$. By the definition of the Vietoris topology, there exists $K_{\alpha}$ such that $K_{\alpha} \cap V \neq \varnothing$ and $K_{\alpha} \cap V_{j} \neq \varnothing$ for $1 \leqq j \leqq n+1$. Choose $q \in K_{\alpha} \cap V$ and $w_{j} \in K_{\alpha} \cap V_{j}$. Then $z_{j}=q w_{j} \in K_{\alpha} \cap U_{j}$ for $j=1, \cdots, n+1$. Now $\left\{z_{1}, \cdots, z_{n+1}\right\}$ is a meet-irredundant set and it is bounded in $K_{\alpha}$ by $q$. This is in contradiction to the hypothesis that $\operatorname{bbr}\left(K_{\alpha}\right) \leqq n$.

Proposition 1.6. Let $S$ be a compact topological semilattice. Then $\{\downarrow s: s \in S\}$ is a closed subset of $P(S)$.

Proof. The set of all singletons $\{\{s\}: s \in S\}$ is homeomorphic to $S$ and hence a compact subset of $P(S)$. Since $\downarrow s=S s$, we have that $\{\downarrow s: s \in S\}$ is simply a translate of the compact set of single- 
tons in the topological semigroup $P(S)$, and hence is compact.

A subset $A$ of a topological space $X$ is said to be locally closed if $A$ is the intersection of an open set and a closed set. Equivalently $A$ is locally closed if it is open in its closure.

Proposition 1.7. Let $S$ be a topological semilattice of finite breadth. Then any dense filter in $S$ is open. Hence every filter in $S$ is locally closed.

Proof. We first assume $S$ has an identity. Let $F$ be a dense filter in $S$. If 1 is not in the interior of $F$, then there exists a net $x_{\alpha}$ in the ideal $I=S \backslash F$ converging to 1 . Since for any $y \in S, y x_{\alpha} \in I$ and $y x_{\alpha}$ converges to $y 1=y, I$ is dense in $S$. Hence by Propositions 1.1 and 1.2, br $(F)<\operatorname{br}(S)$. But by Corollary $1.4 \operatorname{br}(F)=\operatorname{br}(S)$. Hence it must be the case that 1 is in the interior of $F$.

We now drop the assumption that $S$ has an identity and let $F$ be a dense filter in $S$. If $x \in F$ is not in the interior of $F$, then there exists a net $x_{\alpha}$ in the complement of $F$ converging to $x$. Then also the net $y_{\alpha}=x x_{\alpha}$ is not in $F$ and converges to $x$.

Since $F$ is a filter and $x \in F, x F=\downarrow x \cap F$. Since $F$ is dense in $S, x F$ is dense in $x S=\downarrow x$. Hence $\downarrow x \cap F$ is a dense filter in the subsemilattice $\downarrow x$ which has $x$ for an identity. Hence by the first part of the proof $x$ is in the interior of $\downarrow x \cap F$ in $\downarrow x$. But the net $y_{\alpha}$ converges to $x$ in $\downarrow x$ and is not in $\downarrow x \cap F$. This contradiction implies that $x$ must have been in the interior of $F$ in $S$. Hence $F$ is open.

Since any filter in $S$ is a dense filter in its closure, the last statement of the proposition follows from what has just been proved.

We conclude this section with some remarks about compact 0 dimensional semilattices and discrete semilattices. Let $\boldsymbol{S}$ be the category of discrete semilattice monoids and identity preserving semilattice morphisms, and $\boldsymbol{Z}$ the category of compact 0-dimensional semilattice monoids and continuous identity preserving semilattice morphisms. Then, clearly $2=\{0,1\}$, the unique two point semilattice, is both an $\boldsymbol{S}$-object and a $\boldsymbol{Z}$-object. Moreover, as is described at great length in [3], $S$ and $\boldsymbol{Z}$ are dual categories under the functors $D: \boldsymbol{S} \rightarrow \boldsymbol{Z}^{\circ p}$ and $E: \boldsymbol{Z}^{o p} \rightarrow \boldsymbol{S}$ given by $D(\boldsymbol{S})=\boldsymbol{S}(S, 2)(=\widehat{S})$ and $E(T)=Z(T, 2)(=\widehat{T})$, and their obvious extension to the morphisms. Thus, for any $Z$-object $T$, the morphisms $Z(T, 2)$ separate the points, and, in fact, $T \simeq \widehat{T}$.

Definition 1.8. If $T \in Z$, then $k \in T$ is a local minimum in $T$ 
if $\uparrow k$ is open in $T . K(T)$ denotes the set of all local minima of the semilattice $T$.

If $f: T \rightarrow 2$ is a $Z$-morphism then, $f^{-1}(1)$ is a clopen subsemilattice of $T$, and so it has a minimum, $k$. Moreover, since $f^{-1}(1)$ is a filter, $\uparrow k=f^{-1}(1)$. Thus, $f=\chi_{i k}$, the characteristic function of $\uparrow k$. Clearly, $\chi_{i k} \in Z(T, 2)$ for any $k \in K(T)$, and so $\hat{T}=\left\{\chi_{i k}: k \in K(T)\right\}$. Moreover, if $k_{1}, k_{2} \in K(T)$, then $\chi_{i k_{1}} \cdot \chi_{i k_{2}} \in \hat{T}$, and $\chi_{i k_{1}} \cdot \chi_{i k_{2}}=\chi_{i k_{3}}$, where $k_{3}=k_{1} \vee k_{2}$. Thus $(K(T), \vee)$ is a semilattice with 0 as identity, and this semilattice is isomophic to $\hat{T}$.

Conversely, if $S \in S$, then clearly $\chi_{1 s} \in \hat{S}$ for each $s \in S$. However, for $s_{1}, s_{2} \in S, s_{1} \vee s_{2}$ may not be defined in $S$. Thus, there are more semicharacters in $\hat{S}$ than just those generated by some $s \in S$. However, if $f \in \widehat{S}$, then $f^{-1}(1)$ is a filter on $S$, and for $f, g \in \widehat{S}, f \cdot g=\chi_{F}$, where $F=f^{-1}(1) \cap g^{-1}(1)$. Thus, if $(\mathscr{F}(S), \cap)$ is the semilattice of all filters on $S$ under intersection, then $(\mathscr{F}(S), \cap) \simeq \widehat{S}$ (algebraically), and so we topologize $\mathscr{F}(S)$ with the topology from $\hat{S} \subseteq 2^{S}$. Therefore, if $S \in S$, we can refer to $\hat{S}$ as the filter semilattice on $S$.

If $S$ is a compact 0 -dimensional semilattice, then $S^{1}$, the semilattice $S$ with an identity adjoined as an isolated point, is clearly a $Z$-object. Similarly, if $S$ is a discrete semilattice, then $S^{1} \in S$. Moreover, for a semilattice $S$ (discrete or compact 0-dimensional) $\widehat{S}^{1}=\hat{S} \cup\left\{\chi_{\{1\}}\right\}$, so the structure of $\widehat{S}^{1}$ is completely determined by that of $\hat{S}$.

2. Locally compact semilattices with finite breadth. In this section, $S$ is a locally compact semilattice with finite breadth, and $M(S)$ is the Banach algebra of all bounded Borel measures on $S$ under convolution. We will show that for every complex homomorphism $h$ of $M(S)$, there is a filter $F \subset S$ such that $h(\mu)=\mu(F)$ for all $\mu \in M(S)$. Recall that a semicharacter of a semigroup is a homomorphism of the semigroup into the unit disk in $C$ under multiplication. Since the semicharacters of $S$ are precisely the characteristic functions of the filters in $S$, we will have shown that every homomorphism of $M(S)$ is given by integration against a semicharacter.

We begin with a simple measure-theoretic lemma.

Lemma 2.1. Let $\mathscr{F}$ be a family of Borel sets on the locally compact space $X$. Let $\mu$ be a positive bounded Borel measure on $X$. Then $\mu=\mu_{0}+\sum_{n=1}^{\infty} \mu_{n}$, where $\mu_{0}(F)=0$ for all $F \in \mathscr{F}$ and each $\mu_{n}(n \geqq 1)$ is concentrated on some $F_{n} \in \mathscr{F}$. Moreover, $\mu_{0}, \mu_{1}$, $\mu_{2}, \cdots$ are pairwise mutually singular positive bounded Borel measures. Finally, $\mu_{0}$ and $\mu-\mu_{0}$ are uniquely determined.

Proof. Let $l=\sup \{\mu(F) \mid F \in \mathscr{F}\}$; then $l<\infty$. Choose $F_{1} \in$ 
$\mathscr{F} \ni \mu\left(F_{1}\right) \geqq 1 / 2 l$. Let $\mu_{1}=\mu \mid F_{1}$ and $\nu_{1}=\mu \mid F_{1}^{c}$. Let $l_{1}=\sup \left\{v_{1}(F) \mid F \in\right.$ $\mathscr{F}\}$. For each $n>1$ choose $F_{n} \in \mathscr{F} \ni \nu_{n-1}\left(F_{n}\right) \geqq 1 / 2 l_{n-1}$. Let $\mu_{n}=$ $v_{n-1} \mid F_{n}$ and $\nu_{n}=\nu_{n-1} \mid F_{n}^{c}$. Let $l_{n}=\sup \left\{\nu_{n}(F) \mid F \in \mathscr{F}\right\}$. This defines inductively the sequences $\left\{\mu_{n}\right\},\left\{v_{n}\right\}$, and $\left\{l_{n}\right\}$. Note $\mu \geqq \nu_{1} \geqq \nu_{2} \geqq$ $\cdots \geqq \nu_{n} \geqq \nu_{n+1} \geqq \cdots \geqq 0$ and in fact $\mu=\nu_{N}+\sum_{n=1}^{N} \mu_{n}$ for all $N$. We also have $l \geqq l_{1} \geqq l_{2} \geqq \cdots \geqq l_{n} \geqq l_{n+1} \geqq \cdots$ for all $n$. Note that the $\mu_{n}$ are pairwise mutually singular, since if $m<n, \mu_{n} \leqq \nu_{m} \perp \nu_{n}$. Now the $\mu_{n}$ are mutually singular and dominated by $\mu$, so $\sum_{n=1}^{\infty} \mu_{n}$ exists and is dominated by $\mu$. Set $\mu_{0}=\mu-\sum_{n=1}^{\infty} \mu_{n}$. Clearly $\mu_{0} \leqq \nu_{N}$ for all $N$, and so for each $F \in \mathscr{F}, \mu_{0}(F) \leqq \nu_{N}(F) \leqq l_{N} \leqq 2 \nu_{N}\left(F_{N+1}\right)=$ $2\left\|\mu_{N+1}\right\| \rightarrow 0$ as $N \rightarrow \infty$ since $\sum_{n=1}^{\infty}\left\|\mu_{n}\right\|=\left\|\sum_{n=1}^{\infty} \mu_{n}\right\|<\infty$. Thus $\mu_{0}(F)=0$ for all $F \in \mathscr{F}$, and clearly each $\mu_{n}$ is concentrated on $F_{n}$.

It remains to check the uniqueness. Let $L$ be the closed linear span of all bounded Borel measures concentrated on some element of $\mathscr{F}$. Clearly $L$ is an $L$-subspace of $M(X)$ in the sense of [10], and so by the Lebesgue decomposition theorem $M(S)=L \oplus L^{\perp}$, where $L^{\perp}=\{\eta \mid \eta \perp \nu$ for all $\nu \in L\}$. Since $\mu_{0} \in L^{\perp}$ and $\mu-\mu_{0} \in L$, the uniqueness follows.

We now consider a compact semilattice $S$.

Proposition 2.2. Let $S$ be a compact semilattice, and let $\mathscr{F}$ be a family of compact subsets of $S$ such that $\mathscr{F}$ is a closed subset of $P(S)$. If $\mu$ is a probability measure on $S$ such that $\mu(F)=0$ for all $F \in \mathscr{F}$, then there exists a metric quotient $f: S \rightarrow S^{\prime}$ of $S$ such that

$$
\mu\left(f^{-1}(f(F))\right)=0 \text { for all } F \in \mathscr{F} .
$$

Proof. Let $\varepsilon>0$. Suppose for each neighborhood $\mathscr{U}$ of the diagonal $\Delta \subset S \times S$, there exists $F_{\mathscr{U}} \in \mathscr{F}$ such that $\mu\left(\mathscr{U}\left[F_{\mathscr{Z}}\right]\right) \geqq \varepsilon$ where

$$
\mathscr{Q}[A]=\{b \in S:(b, a) \in \mathscr{U} \text { for some } a \in A\} .
$$

Since $\mathscr{F}$ is closed and hence compact in $P(S)$, some subnet $F_{\alpha}$ of the net $\left\{F_{\mathscr{U}}: \Delta \subset\right.$ interior $\left.(\mathscr{U})\right\}$ converges to $F \in \mathscr{F}$. Since $\mu(F)=0$, by outer regularity there exists an entourage $\mathscr{U}$ (i.e., a neighborhood of the diagonal) such that $\mu(\mathscr{\mathscr { C }}[F])<\varepsilon$. Pick an entourage $\mathscr{V}$ such that $\mathscr{V} \circ \mathscr{V} \subset \mathscr{Q}$ and $\mathscr{V}$ is symmetric. Since $F_{\alpha} \rightarrow F$, there exists a $\beta$ such that $F_{\beta} \subset \mathscr{C}[F]$ and $\mu\left(\mathscr{C}\left[F_{\beta}\right]\right) \geqq \varepsilon$. Then

$$
\varepsilon \leqq \mu\left(\mathscr{V}\left[F_{\beta}\right]\right) \leqq \mu(\mathscr{V} \circ \mathscr{V}[F]) \leqq \mu(\mathscr{U}[F])<\varepsilon,
$$

a contradiction. Hence there exists an entourage $\mathscr{U}_{\varepsilon}$ such that $\mu\left(\mathscr{U}_{\varepsilon}[F]\right)<\varepsilon$ for all $F \in \mathscr{F}$. 
Now using the uniform continuity of multiplication on $S$, we choose inductively for each $n$ a compact entourage $\mathscr{U}_{n}$ satisfying

(1) $\Delta \wedge \mathscr{U}_{n} \subset \mathscr{U}_{n}$ (products taken coordinatewise),

(2) $\mathscr{Q}_{n}=\mathscr{U}_{n}^{-1}$,

(3) $\mu\left(\mathscr{U}_{n}(F)\right)<1 / n$ for all $F \in \mathscr{F}$,

(4) $\mathscr{U}_{n} \circ \mathscr{U}_{n} \subset \mathscr{U}_{n-1}$.

It is now standard that $\rho=\cap\left\{\mathscr{C}_{n}: n \in \omega\right\}$ is a closed congruence on $S$ (see e.g. [4], Proposition 8.6, p. 49) and $S^{\prime}=S / \rho$ is metrizable. That $\mu\left(f^{-1}(f(F))\right)=0$ for all $F \in \mathscr{F}$ follows easily from property (3).

Proposition 2.3. Let $S$ be a compact semilattice of finite breadth. Suppose $h$ is a complex homomorphism on $M(S)$ which annihilates the discrete measures. Then $h=0$.

Proof. Clearly it suffices to show $h(\mu)=0$ for every probability measure $\mu$. Let $n=\operatorname{bbr}(S)$. We argue by induction on $n$, and clearly it holds for $n=0$. We show that if the proposition is valid for $\operatorname{bbr}(S)<n$, it is true for $\operatorname{bbr}(S)=n$. Let $\mu$ be a probability measure on $S$. Let $\mathscr{F}_{1}$ denote the collection of principal ideals $\downarrow s$, $s \in S$, let $\mathscr{F}_{2}$ denote the collection of compact subsemilattices of $b b r \leqq n-1$, and let $\mathscr{F}=\mathscr{F}_{1} \cup \mathscr{F}_{2}$. By $2.1, \mu=\mu_{0}+\sum_{k=1}^{\infty} \mu_{k}$ where $\mu_{0}$ annihilates every member of $\mathscr{F}$ and each $\mu_{k}, k \geqq 1$, is concentrated on some member of $\mathscr{F}$.

If $\mu_{k}$ is concentrated on some member of $\mathscr{F}_{2}$, our inductive hypothesis gives $h\left(\mu_{k}\right)=0$. If $\mu_{k}$ is concentrated on $\downarrow x_{k}$, then $\mu_{k}=\mu_{k} * \delta_{x_{k}}$ (the unit point mass at $\left.x_{k}\right)$, so $h\left(\mu_{k}\right)=h\left(\mu_{k} * \delta_{x_{k}}\right)=h\left(\mu_{k}\right)$. $0=0$. Thus we need only show that $h\left(\mu_{0}\right)=0$, and so we assume without loss of generality that $\mu$ annihilates every member of $\mathscr{F}$.

By 2.1 we can write the convolution power $\mu^{n+2}=\nu_{0}+\nu_{1}$ where $\nu_{0}$ annihilates every member of $\mathscr{F}$ and $\nu_{1}$ lives on a countable union of members of $\mathscr{F}$.

By Propositions 1.5 and $1.6 \mathscr{F}_{1}, \mathscr{F}_{2}$ and hence $\mathscr{F}$ are closed in $P(S)$. Hence by 2.2 there are closed congruences $\rho_{1}$ and $\rho_{2}$ on $S$ such that $S_{1}=S / \rho_{1}$ and $S_{2}=S / \rho_{2}$ are metrizable semilattices and $\mu\left(f_{1}^{-1}\left(f_{1}(F)\right)\right)=0$ for all $F \in \mathscr{F}_{1}, \nu_{0}\left(f_{2}^{-1}\left(f_{2}(F)\right)\right)=0$ for all $F \in \mathscr{F}_{2}$, where $f_{1}: S \rightarrow S_{1}$ and $f_{2}: S \rightarrow S_{2}$. Let $\rho=\rho_{1} \cap \rho_{2}$, and $f: S \rightarrow S^{\prime}=S / \rho$.

Since $S^{\prime}$ embeds as a subdirect product of $S_{1} \times S_{2}, S^{\prime}$ is metrizable. Furthermore since $\rho \subset \rho_{1}$ and $\rho \subset \rho_{2}, \mu\left(f^{-1}(f(F))\right)=0$ for all $F \in \mathscr{F}$ and $\nu_{0}\left(f^{-1}(f(F))\right)=0$ for all $F \in \mathscr{F}$.

Choose a countable dense set $\left\{y_{k}\right\} \subset S^{\prime}$; we have $I^{\prime}=\bigcup_{k} \downarrow y_{k}$ is a dense Borel ideal of $S^{\prime}$. We have for $y \in S^{\prime}$ and the induced measure $f(\mu)$ on $S^{\prime}$ that

$$
f(\mu)(\downarrow y)=\mu\left(f^{-1}(\downarrow y)\right)=\mu f^{-1}(f(\downarrow x))=0 \text { where } \quad f(x)=y .
$$


Similarly $f\left(\nu_{0}\right)(\downarrow y)=0$. Hence $f(\mu)\left(I^{\prime}\right)=0=f\left(\nu_{0}\right)\left(I^{\prime}\right)$. Thus $I=f^{-1}\left(I^{\prime}\right)$ is a Borel ideal of $S$ such that $\mu(I)=f(\mu)\left(I^{\prime}\right)=0=f\left(\nu_{0}\right)\left(I^{\prime}\right)=\nu_{0}(I)$. Hence $\mu=\mu \mid(S \backslash I)$ and $\nu_{0}=\nu_{0} \mid(S \backslash I)$.

Let $R=S \backslash I$. If $T$ is a compact subsemilattice of $S$ contained in $R$, we claim $\mu(T)=0$.

Note first of all that $f(T)=f\left(f^{-1}(f(T)) \cap I^{*}\right)$. One containment is obvious. Conversely let $y=f(t) \in f(T)$ where $t \in T$. Since $I^{\prime}=f(I)$ is dense in $S^{\prime}$, there exists a net $\left\{y_{\alpha}\right\} \subset I^{\prime}$ converging to $y$ in $S^{\prime}$. Pick $x_{\alpha} \in I$ such that $f\left(x_{\alpha}\right)=y_{\alpha}$. By compactness some subnet of $x_{\alpha}$ converges to $x \in I^{*}$. By continuity $f\left(x_{\alpha}\right)$ converges to $f(x)=y$. Hence $x \in f^{-1}(f(T)) \cap I^{*}$ and so $y \in f\left(f^{-1}(f(T)) \cap I^{*}\right)$.

Now $f^{-1}(f(T)) \cap I^{*}=P$ is a subsemilattice of $S$ contained in $I^{*} \backslash I$ (since $T \cap I=\varnothing$ ). By Proposition 1.2 bbr $(P) \leqq n-1$. Hence $P \in \mathscr{F}$, and thus $\mu(P)=0$. Hence by the way $S^{\prime}$ was chosen $\mu\left(f^{-1}(f(P))\right)=0$. But $f^{-1}(f(P))=f^{-1} f\left(f^{-1}(f(T)) \cap I^{*}\right)=f^{-1} f(T) \supset T$. Hence $\mu(T)=0$. Thus the claim is completed. Note in particular if $x \in S \backslash I$, then $\mu(\uparrow x)=0$.

Now we claim $\mu^{n+2}(R)=0$. In fact, for $1 \leqq i \leqq n+2$, let $E_{i}=$ $\left\{\left(x_{1}, \cdots, x_{n+2}\right) \in R^{n+2} \mid x_{i} \geqq x_{1} \cdots \hat{x}_{i} \cdots x_{n+2} \in R\right\}$, and let $F=\left\{\left(x_{1}, \cdots, x_{n+1}\right) \in\right.$ $\left.R^{n+1} \mid x_{1} \cdots x_{n+1} \in R\right\}$. (Here $\hat{x}_{i}$ means $x_{i}$ is to be omitted.) Then using the Fubini Theorem we have

$$
\begin{aligned}
(\mu \times & \cdots \times \mu)\left(E_{i}\right)=\int_{R^{n+2}} \chi_{E_{i}}\left(x_{1}, \cdots, x_{n+2}\right) d(\mu \times \cdots \times \mu)\left(x_{1}, \cdots, x_{n+2}\right) \\
& =\int_{R^{n+1}} \int_{R} \chi_{E_{i}}\left(x_{1}, \cdots, x_{n+2}\right) d \mu\left(x_{i}\right) d(\mu \times \cdots \times \mu)\left(x_{1}, \cdots, \hat{x}_{i}, \cdots, x_{n+2}\right) \\
& =\int_{F} \int_{R} \chi_{E_{i}}\left(x_{1}, \cdots, x_{n+2}\right) d \mu\left(x_{i}\right) d \mu \times \cdots \times \mu\left(x_{1}, \cdots, \hat{x}_{i}, \cdots, x_{n+2}\right) \\
& =\int_{F} \int_{R} \chi_{x_{1} \cdots \hat{x}_{i} \cdots x_{n+2}}\left(x_{i}\right) d \mu\left(x_{i}\right) d(\mu \times \cdots \times \mu)\left(x_{1}, \cdots, \hat{x}_{i}, \cdots, x_{n+2}\right) \\
& =\int_{F} \mu\left(\uparrow x_{1} \cdots \hat{x}_{i} \cdots x_{n+2}\right) d(\mu \times \cdots \times \mu)\left(x_{1}, \cdots, \hat{x}_{i}, \cdots, x_{n+2}\right)=0 .
\end{aligned}
$$

Since $\operatorname{bbr}(S)=n, \operatorname{br}(S) \leqq n+1$ and so $\left\{\left(x_{1}, \cdots, x_{n+1}\right) \in R^{n+2} \mid x_{1} \cdots x_{n+2} \in R\right\} \subset$ $\bigcup_{i=1}^{n+2} E_{i}$. Thus $\left.\mu^{n+2}(R)=(\mu \times \cdots \times \mu)\left(\left\{x_{1}, \cdots, x_{n+2}\right) \in R^{n+2} \mid x_{1} \cdots x_{n+2} \in R\right\}\right) \leqq$ $(\mu \times \cdots \times \mu)\left(\bigcup_{i=1}^{n+2} E_{i}\right)=0$. So $\mu^{n+2}$ is concentrated on $I$. But $\nu_{0}(I)=0$ and so $\mu^{n+2}=\nu_{1}$; i.e., $\mu^{n+2}$ lives on a countable union of elements of $\mathscr{F}$. It follows that $h\left(\mu^{n+2}\right)=0$, whence $h(\mu)=0$.

Proposition 2.4. Let $S$ be a locally compact semilattice of finite breadth and suppose $h$ is a complex homomorphism of $M(S)$ which annihilates the discrete measures. Then $h=0$.

Proof. We assume without loss of generality that $\mu$ is a probability measure on $S$, and show $h(\mu)=0$. Let $\varepsilon>0$, and choose a 
compact set $K \subset S$ such that $\mu(S \backslash K)<\varepsilon$. If $n$ is the breadth of $S, K^{n}$ is a compact subsemilattice of $S$ and $\mu\left(S \backslash K^{n}\right)<\varepsilon$. By 2.3 , since $h$ annihilates every discrete measure in $M\left(K^{n}\right), h\left(\mu \mid K^{n}\right)=0$. So $|h(\mu)|=$ $\left|h\left(\mu \mid S \backslash K^{n}\right)\right| \leqq\left\|\mu \mid S \backslash K^{n}\right\|<\varepsilon$. Since $\varepsilon>0$ is arbitrary, $h(\mu)=0$.

THEOREM 2.5. Let $S$ be a locally compact semilattice of finite breadth. Let $h \in \Delta M(S)$. Let $F_{h}=\left\{s \in S \mid h\left(\delta_{s}\right)=1\right\}$. Then $F_{h}$ is a locally closed (hence Borel) filter, and for all $\mu \in M(S), h(\mu)=\mu\left(F_{h}\right)$.

Proof. Clearly $F_{h}$ is a filter. By $1.7, F_{h}$ is locally closed, and hence Borel. We observe first that if $\mu \in M(S), h(\mu)=h\left(\mu \mid F_{h}\right)$. Let $I_{h}=S \backslash F_{h}$, and let $K \subset I_{h}$ be an arbitrary compact subsemilattice. Then $h$ annihilates every discrete measure living on $K$ (since for $x \in K, h\left(\delta_{x}\right)=0$ ) and so by $2.3, h(\mu \mid K)=0$. Using the regularity of $\mu$ we see that $h\left(\mu \mid I_{h}\right)=0$. Thus $h(\mu)=h\left(\mu \mid F_{h}\right)$.

Now let $\varepsilon>0$ and assume without loss of generality that $\mu$ is positive. Choose $K$ a compact subset of $F_{h}$ such that $\mu\left(F_{h} \backslash K\right)<\varepsilon$. Then if $n=b r(S), K^{n}$ is a compact subsemilattice of $F_{h}$ and $\mu\left(F_{h} \backslash K^{n}\right)<\varepsilon$. Let $k=\wedge K^{n}$. Then $k \in K^{n}$ and $h\left(\mu \mid K^{n}\right)=h\left(\delta_{k}\right) h\left(\mu \mid K^{n}\right)=$ $h\left(\delta_{k} * \mu \mid K^{n}\right)=h\left(\mu\left(K^{n}\right) \delta_{k}\right)=\mu\left(K^{n}\right) h\left(\delta_{k}\right)=\mu\left(K^{n}\right)$. Hence $\left|h(\mu)-\mu\left(F_{h}\right)\right| \leqq$ $\left|h(\mu)-h\left(\mu \mid F_{h}\right)\right|+\left|h\left(\mu \mid F_{h}\right)-h\left(\mu \mid K^{n}\right)\right|+\left|h\left(\mu \mid K^{n}\right)-\mu\left(K^{n}\right)\right|+\mid \mu\left(K^{n}\right)-$ $\mu\left(F_{h}\right) \mid \leqq 2 \mu\left(F_{h} \backslash K^{n}\right)<2 \varepsilon$. Since $\varepsilon>0$ is arbitrary, we have $h(\mu)=$ $\mu\left(F_{h}\right)$.

If $S$ is a locally compact semilattice of finite breadth, then so is $S^{1}$. Moreover, for any locally compact semigroup $S, M\left(S^{1}\right) \simeq$ $M(S) \oplus C$, and so $\Delta M\left(S^{1}\right) \simeq \Delta M(S) \cup\{0\}$, where 0 is the 0 -homomorphism of $M(S)$, and $\Delta M\left(S^{1}\right)$ is the one-point compactification of $\Delta M(S)$. Thus, if we determine the structure of $\Delta M\left(S^{1}\right)$, we have also determined the structure of $\Delta M(S)$, and conversely. The difference is that $\Delta M\left(S^{1}\right)$ is always a semigroup [10], whereas this is not true if $S$ has no identity. Thus, throughout the rest of this section, we assume $S$ is a locally compact semilattice with identity having finite breadth.

If $S$ is such a semilattice, then according to Theorem 2.5, each complex homomorphism $h$ of $M(S)$ is given by integration over some filter $F \leqq S$. Thus, $\Delta M(S)$ is $\mathscr{F}(S)$, the set of all filters on $S$. Moreover, it is clear that the product of two homomorphisms of $M(S)$ corresponds to the intersection of their associated filters. Hence, algebraically, $\Delta M(S) \simeq(\mathscr{F}(S), \cap)$. Now, $\Delta M(S)$ is a semitopological semilattice in the weak topology [10]. But $\Delta M(S)$ is also compact and a semilattice, and so $\Delta M(S)$ is a compact topological semilattice in the weak topology [8].

From our discussion at the end of $\S 1$, we know that $(\mathscr{F}(S), \cap) \simeq$ 
$\widehat{S}_{d}$ is a compact 0-dimensional topological semilattice, where $S_{d}$ is the semilattice $S$ with the discrete topology. But, the topology on a compact semilattice is uniquely determined by the algebraic structure [7]. Hence, $\Delta M(S) \simeq \hat{S}_{d}$.

One of the key results in Taylor's work is the determination that, for any convolution measure algebra $M$, the so-called critical points in $\Delta M$ carry the cohomology of $\Delta M$. A critical point is an element $x \in \Delta M$ such that $\uparrow x$ is open and $x \geqq 0$. For the semilattice $\Delta M(S)$, a critical point is what we referred to in $\S 1$ as a local minimum. Hence the critical points of $\Delta M(S)$ are $K(\Delta M(S))=$ $K\left(\widehat{S}_{d}\right)=K(\mathscr{F}(S), \cap)$. However, the critical points of $\mathscr{F}(S)$ are precisely the principal filters on $S$, i.e., the filters $F \subset S$ of the form $F=\uparrow s$ for some $s \in S$ [3]. Hence, $K(\Delta M(S))=\left\{h: h^{-1}(1)=\uparrow s\right.$ for some $s \in S\}$ is the set of critical points of $\Delta M(S)$. Identifying $s$ with the principal filter $\uparrow s$ we have a natural correspondence between $S$ and the critical points of $\Delta M$.

For a semisimple convolution measure algebra $M$, Taylor defines the structure semigroup $T$ of $M$ to be the unique compact abelian monoid $T$ such that there is an isometric $L$-isomorphism $f: M \rightarrow M(T)$ such that $f(M)$ is weak *-dense in $M(T)$, each complex homomorphism on $M$ is given by integration against some semicharacter $h \in \hat{T}$, and $\hat{T}$ separates the points of $T$. In general, knowing the algebraic semigroup $\hat{T}$ does not determine the semigroup $T$ uniquely. If we return now to the situation where $M=M(S)$ for some locally compact semilattice $S$ with identity and having finite breadth, then $\Delta M(S)$ is a semilattice, and Taylor's work shows that the structure semigroup for $M(S)$ has the discrete semigroup $\Delta M(S)$ as its semilattice of semicharacters. Since the structure semigroup $T$ for $M(S)$ always has enough semicharacters to separate points and since in this case each semicharacter is idempotent, it follows that $T$ must be idempotent and hence a semilattice. Since the semicharacters of a semilattice have range $\{0,1\}, T$ can be embedded in a product of the two-point semilattice and hence must be totally disconnected. According to the duality between $S$ and $Z$ discussed in $\S 1$, we must then have that $T \simeq \Delta \widehat{M(S)})_{d}$, where again $\Delta M(S)_{d}$ is the discrete semilattice $\Delta M(S)$. But, we know $\Delta M(S) \simeq \widehat{S}_{d}$, so we conclude $T \simeq \widehat{\left(\hat{S}_{d}\right)_{d}}$. We summarize our results in the following:

THEOREM 2.6. Let $S$ be a compact semilattice with identity and having finite breadth. Then $\Delta M(S) \simeq \widehat{S}_{d}$ is a compact 0-dimensional semilattice, and the critical points of $\Delta M(S)$ are precisely those complex homomorphisms $h$ of $M(S)$ of the form $h=\chi_{1 s}$ for some $s \in S$. Moreover, the structure semilattice of $M(S)$ is $\widehat{\left(\hat{S}_{d}\right)_{d}}$. 
As we remarked above, Taylor shows that $H^{*}(\Delta M(S))$ is the direct sum of the cohomology of the maximal subgroups $H(e)$ as $e$ ranges over the critical points of $\Delta M(S)$. However, since $\triangle M(S)$ is a semilattice, $H(e)=\{e\}$ for all $e \in \Delta M(S)$, so $H^{*}(\Delta M(S)) \simeq \bigoplus_{s \in S_{d}} H(\{s\})$, where $S_{d}$ represents the critical points of $\Delta M(S)$. We draw two conclusions.

First, the Shilov Idempotent Theorem states that the idempotents in $M(S)$ are in one-to-one correspondence with $H^{\circ}(\Delta M(S)) \simeq$ $\bigoplus_{s \in S_{d}} H^{0}(\{s\})$. Hence, since the correspondence is given by the Gelfand transform, so that $\hat{\sigma}_{s} \sim H^{0}(\{s\}) \forall s \in S$, we conclude that $\mu \in M(S)$ is idempotent if and only if $\mu=\sum_{k=1}^{n} \alpha_{k} \delta_{s_{k}}$ for some $s_{1}, \cdots, s_{n} \in S$, where $(\forall s \in S) \sum_{s \leq s_{k}} \alpha_{k}=\left\{\begin{array}{l}0 \\ 1\end{array}\right.$ (this latter follows from the fact that $\mu(\uparrow s) \in\{0,1\}$ as $\mu(\uparrow s)=h(\mu)$ where $\left.h=\chi_{i s} \in \Delta M(S)\right)$.

Our second conclusion is as follows. Since $\Delta M(S)$ is 0-dimensional, $H^{n}(\Delta M(S))=0$ for $n \geqq 1$, so that, according to the ArensRoyden theorem, the group of invertible elements in $M(S)$ is precisely the group of exponential measures.

3. P-algebras. In [9], Newman defines a $P$-algebra to be a semisimple convolution measure algebra $M$ such that whenever $\mu$ is positive element of $M$ and $h \in \Delta M$, the $h(\mu) \geqq 0$. He shows (Theorem 1 of [9]) that these are precisely the semisimple convolution measure algebras whose structure semigroups are in fact semilattices. It is easily checked that the equivalence $(1) \Leftrightarrow(2) \Leftrightarrow(3)$ of Theorem 1 of [9] is true without assuming semisimplicity, so we shall define a $P$ algebra to be a convolution measure algebra $M$ such that $h(\mu) \geqq 0$ for all $h \in \Delta M$ and all $\mu \in M$ such that $\mu \geqq 0$. Thus we have shown (Theorem 2.5) that if $S$ is a locally compact semilattice with finite breadth, then $M(S)$ is a $P$-algebra. We shall see that this is true in a somewhat more general setting, but first we give a general condition which insures that $M(S)$ is a semisimple convolution measure algebra.

DEFINITION 3.1. A locally compact semilattice is said to be Lawson if it has a neighborhood basis of compact subsemilattices. Equivalently, $S$ is Lawson if the semilattice homomorphisms into $([0,1], \wedge)$ separate the points of $S$. (See [6].)

THEOREM 3.2. If $S$ is Lawson, then $M(S)$ is semisimple.

Proof. Let $I=([0,1], \wedge)$. Baartz showed in [1] that if $n<\infty$, then $M\left(I^{n}\right)$ is semisimple. Now if $\alpha$ is any cardinal number, $M\left(I^{\alpha}\right)=$ proj $\lim _{n<\infty} M\left(I^{n}\right)$, where the maps $M\left(I^{\alpha}\right) \rightarrow M\left(I^{n}\right)$ are quotient maps, 
and where a measure $\mu \in M\left(I^{\alpha}\right)$ is zero iff its image in every $M\left(I^{n}\right)$ is zero. Since the complex homomorphisms of each $M\left(I^{n}\right)$ separate the points, it follows that the same is true of $M\left(I^{\alpha}\right)$.

Now clearly if $S$ is a Lawson semilattice there is a continuous, injective semilattice morphism $f: S \rightarrow I^{\alpha}$ for some cardinal $\alpha$. Every nonzero measure $\mu$ in $M(S)$ lives on a $\sigma$ compact set $T_{\mu}$, and $f: T_{\mu} \rightarrow$ $f\left(T_{\mu}\right)$ is a Borel isomorphism. In particular $f(\mu) \neq 0$. So $f: M(S) \rightarrow$ $M\left(I^{\alpha}\right)$ is an injection, and it follows that $M(S)$ is semisimple.

COROLlary 3.3. If $S$ is a locally compact semilattice with finite breadth, then $M(S)$ is a semisimple P-algebra.

Proof. Immediate from 2.5, 3.2, and the observation that $S$ must be Lawson.

We can actually assert that $M(S)$ is a $P$-algebra for somewhat more general $S$.

THEOREM 3.4. Let $S$ be a locally compact semilattice. Suppose every $\mu \in M(S)$ is concentrated on a Borel set which is the countable union of compact subsemilattices of finite breadth. Then $M(S)$ is a P-algebra.

Note. We are not asserting here that $M(S)$ is semisimple.

Proof. Straightforward from 2.5.

Here are two examples of compact finite breadth semilattices which cannot be imbedded in finite dimensional cubes and are thus not dealt with by Newman's methods, and a third to show that semilattices with nonfinite breadth, but still satisfying the hypotheses of Theorem 3.4, do exist.

EXAMPLE 3.5 (cf. Exercise 1.12 of [2]). Let $S$ be the Rees quotient $I^{2} /(I \times\{0\} \cup\{0\} \times I) . \quad S$ has breadth 2 .

EXAmPLE 3.6. Let $S=\left\{\left(x_{n}\right) \in I^{\infty} \mid x_{n}=0\right.$ for all but at most one $n$ ). Then $S$ has breadth 2 , but cannot be imbedded in a finitedimensional cube $I^{n}$. In fact, $S$ contains an infinite set $\left\{x_{n}\right\}$ of elements which annihilate each other pair-wise, and for any finite $n$, it is not difficult to see that no such infinite sets exist in $I^{n}$, (no matter what element of $I^{n}$ is the image of the 0 in $S$ ).

EXAMPLE 3.7 . For each $n \geqq 1$, let $I^{n}$ denote the $n$-fold product 
of the semilattice $([0,1], \wedge)$ with itself. Let $S_{1}=\bigcup_{n \geqq 1} I^{n}$, and let $S=S_{1} / R$, where $R$ is the relation on $S_{1}$ which identifies the identity of $I^{n}$ with the zero of $I^{n+1}$ for each $n=1,2, \cdots$. Then $S$ is a locally compact semilattice satisfying the hypotheses of Theorem 3.4 , but $\operatorname{br}(S)=\infty$. If $S^{1}$ denotes $S$ with an identity added as the one point compactification of $S$, then $S$ is a compact semilattice with identity satisfying the hypotheses of 3.4 which fails to have finite breadth.

We remark that the investigations of this paper can be carried out in a more general setting. Let $S$ be a locally compact semilattice, and let $N(S)$ be the norm closure in $M(S)$ of all measures with support a subset of a compact finite breadth subsemilattice of $S$. Then $N(S)$ is a norm-closed $L$-subalgebra of $M(S)$ which consists precisely of all measures in $M(S)$ which vanish outside of a countable union of compact semilattices with finite breadth.

THEOREM 3.8. Let $S$ be a locally compact semilattice, let $h \in \Delta N(S)$, and let $F_{h}=\left\{s \in S: h\left(\delta_{s}\right)=1\right\}$. Then for all $\mu \in N(S)$, $h(\mu)=\mu\left(F_{h} \cap K\right)$ where $K$ is any countable union of compact subsemilattices of finite breadth such that $\mu$ vanishes outside of $K$.

Proof. Let $\mu \in N(S)$ which vanishes outside of $K=\bigcup_{j=1}^{\infty} K_{j}$ where each $K_{j}$ is a compact subsemilattice of finite breadth. We may assume the $K_{j}$ tower up (by defining a new sequence with $n$th element $K_{1} \cdot K_{2} \cdots \cdot K_{n}$ if necessary). Let $\mu_{j}=\mu \mid K_{j}$. Then $\left\{\mu_{j}\right\}$ norm converges to $\mu$. By Theorem 2.5 we have $h\left(\mu_{j}\right)=\mu\left(F_{h} \cap K_{j}\right)$ for each $j$. Hence $h(\mu)=\lim h\left(\mu_{j}\right)=\lim \mu\left(F_{h} \cap K_{j}\right)=\mu\left(F_{h} \cap K\right)$.

Using this theorem we deduce as before that $\Delta(N(S)) \cong \widehat{S}_{d}$ and the structure semigroup $T$ of $N(S)$ is $\left(\widehat{\left.\hat{S}_{d}\right)_{d}}\right.$. Other analogous remarks that were made about $M(S)$ before can now be made about $N(S)$.

In general any filter $F$ on a locally compact semilattice $S$ gives rise to a homomorphism $h_{F} \in \Delta(M(S))$ defined by $h_{F}(\mu)$ is the inner $\mu$ measure of $F$. Hence $\widehat{S}_{d}$ always sits in $\Delta(M(S))$. It is conjectural that $\hat{S}_{d}$ is $\Delta(M(S)$ if and only if $M(S)=N(S)$. (We have shown the "if" direction.)

\section{REFERENCES}

1. A. Baartz, The measure algebra of a locally compact semigroup, Pacific J. Math., 21 (1967), 199-214.

2. K. H. Hofmann and M. Mislove, Epics of compact Lawson semilattices are surjective, Archiv der Math., 26 (1975), 337-345.

3. K. H. Hofmann, M. Mislove, and A.R. Stralka, The Pontryagin duality of compact 0-dimensional semilattices and its applications, Lecture Notes in Mathematics 396, Springer-Verlag, Heidelberg, 1974. 
4. K. H. Hofmann and P. Mostert, Elements of Compact Semigroups, Merrill, Columbus, Ohio, 1966.

5. K. H. Hofmann and A. Stralka, The algebraic theory of compact Lawson semilattices, Dissertationes Math., 137 (1976), 53 pp.

6. J. D. Lawson, Topological semilattices small with semilattices, J. London Math. Soc., I (1969), 719-724.

7. - Intrinsic topologies in topological lattices and semilattices, Pacific J. Math., 44 (1973), 593-102.

8. —_ Additional notes on continuity in semitopological semigroups, Semigroup Forum, to appear.

9. S. E. Newman, Measure algebras on idempotent semigroups, Pacific J. Math., 31 (1969), 161-169.

10. J. L. Taylor, Measure Algebras, CBMS Regional Conference Series in Mathematics 16, A. M. S., Providence, 1972.

Received June 24, 1976. Research by the first author was partially supported by NSF contract MPS 73-08812. Research by the remaining two authors was partially supported by NSF contract MPS 71-02871-A04.

LoUisiana State University

AND

Tulane University 



\section{PACIFIC JOURNAL OF MATHEMATICS}

EDITORS

RICHARD ARENS (Managing Editor)

University of California

Los Angeles, California 90024

R. A. Beaumont

University of Washington

Seattle, Washington 98105
J. DUGUNDJI

Department of Mathematics

University of Southern Californı

Los Angeles, California 90007

D. Gilbarg and J. Milgram

Stanford University

Stanford, California 94305

\section{ASSOCIATE EDITORS}

E. F. BECKENBACH

B. H. NeumanN

F. WOLF

K. YoSHIDA

\section{SUPPORTING INSTITUTIONS}

UNIVERSITY OF BRITISH COLUMBIA

CALIFORNIA INSTITUTE OF TECHNOLOGY

UNIVERSITY OF CALIFORNIA

MONTANA STATE UNIVERSITY

UNIVERSITY OF NEVADA

NEW MEXICO STATE UNIVERSITY

OREGON STATE UNIVERSITY

UNIVERSITY OF OREGON

OSAKA UNIVERSITY
UNIVERSITY OF SOUTHERN CALIFORNIA

STANFORD UNIVERSITY

UNIVERSITY OF TOKYO

UNIVERSITY OF UTAH

WASHINGTON STATE UNIVERSITY

UNIVERSITY OF WASHINGTON

AMERICAN MATHEMATICAL SOCIETY

NAVAL WEAPONS CENTER 


\section{Pacific Journal of Mathematics}

Vol. 69 , No. 1

May, 1977

V. V. Anh and P. D. Tuan, On starlikeness and convexity of certain analytic

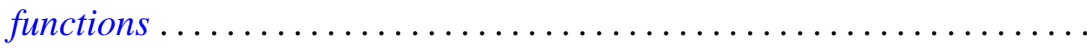

Willard Ellis Baxter and L. A. Casciotti, Rings with involution and the prime

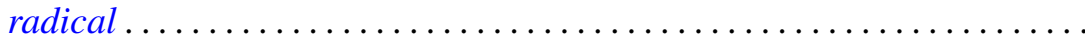

Manuel Phillip Berriozabal, Hon-Fei Lai and Dix Hayes Pettey,

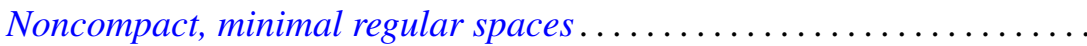

Sun Man Chang, Measures with continuous image law ................

John Benjamin Friedlander, Certain hypotheses concerning

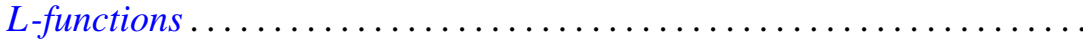

Moshe Goldberg and Ernst Gabor Straus, On characterizations and

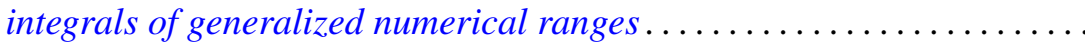

Pierre A. Grillet, On subdirectly irreducible commutative semigroups ...... 55

Robert E. Hartwig and Jiang Luh, On finite regular rings ..............

Roger Hugh Hunter, Fred Richman and Elbert A. Walker, Finite direct sums of cyclic valuated p-groups ........................... 97

Atsushi Inoue, On a class of unbounded operator algebras. III ......... 105

Wells Johnson and Kevin J. Mitchell, Symmetries for sums of the Legendre

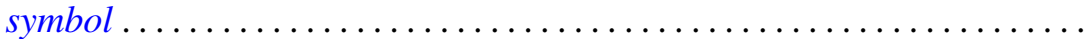

Jimmie Don Lawson, John Robie Liukkonen and Michael William Mislove,

Measure algebras of semilattices with finite breadth

Glenn Richard Luecke, A note on spectral continuity and on spectral properties of essentially $G_{1}$ operators ...............

Takahiko Nakazi, Invariant subspaces of weak-* Dirichlet algebras . .

James William Pendergrass, Calculations of the Schur group ...

Carl Pomerance, On composite $n$ for which $\varphi(n) \mid n-1$. II. . .

Marc Aristide Rieffel and Alfons Van Daele, A bounded operator approach to Tomita-Takesaki theory........................

Daniel Byron Shapiro, Spaces of similarities. IV. $(s, t)$-families ...

Leon M. Simon, Equations of mean curvature type in 2 independent variables.

Joseph Nicholas Simone, Metric components of continuous images of ordered compacta ............................

William Charles Waterhouse, Pairs of symmetric bilinear forms in

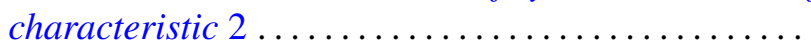

